\title{
Microlensing neutron stars
}

\author{
D. J. Schwarz ${ }^{1,2}$ and D. Seidel ${ }^{2}$ \\ 1 Institut für Theoretische Physik, Technische Universität Wien, Wiedner Hauptstraße 8-10, 1040 Wien, Austria \\ e-mail: dschwarz@hep.itp.tuwien.ac.at \\ 2 Institut für Theoretische Physik, J. W. Goethe-Universität, 60054 Frankfurt am Main, Germany \\ e-mail: seidel@th.physik.uni-frankfurt.de
}

Received 27 March 2001 / Accepted 28 March 2002

\begin{abstract}
We investigate the chances that neutron stars act as the lens in a gravitational microlensing event towards the galactic bulge or a spiral arm. The observation of neutron stars by means of gravitational microlensing would allow the estimation of neutron star masses independently of the property of being a pulsar in a binary system. We estimate the contribution of neutron stars to the optical depth and the lensing rate based on two different models of pulsar distribution in the galaxy. Since only a small fraction of all neutron stars are pulsars, it is unlikely to find a pulsar that acts as a microlens by chance. A position comparison of known radio pulsars with observed microlensing candidates towards the galactic bulge and spiral arms shows no candidate pairs, which is consistent with the theoretical expectation. To improve the probability of microlensing a pulsar, we suggest a search for gravitational microlensing events of known nearby high proper motion pulsars. The pulsar PSR J1932+1059 is a good candidate for an astrometric detection of gravitational lensing.
\end{abstract}

Key words. gravitational lensing - stars: neutron - stars: pulsars: individual: PSR J1932+1059

\section{Introduction}

The determination of neutron star masses so far has been limited to binary systems. Particularly, the masses of radio pulsars in binary systems lie in an impressively narrow range $M_{\mathrm{PSR}}=1.35 \pm 0.04 M_{\odot}$ (Thorsett et al. 1993; Thorsett \& Chakrabarty 1999). The spread of masses based on the measurement of X-ray binary systems is somewhat larger (Nagase 1989; Reynolds et al. 1992; Heap \& Corcoran 1992; Reynolds et al. 1997; Ash et al. 1999), but the measurements are consistent with the mass value quoted above. However, it is not clear whether these measurements are representative of all neutron stars. In this paper we investigate the chances of estimating the mass of neutron stars by gravitational microlensing.

The galaxy hosts $\sim 10^{9}$ neutron stars (Timmes et al. 1996), of which $\sim 10^{5}$ are pulsars (Narayan 1987). Thus the fraction of neutron stars which are also pulsars is only $10^{-3}-10^{-4}$. Moreover, only a small fraction of pulsars appears to be in binary systems (of $\sim 10^{3}$ known radio pulsars, only $\sim 10^{2}$ are known to be in binary systems). The range of observed masses is surprisingly narrow in view of the theoretical stability considerations, which allow neutron star masses in the range $0.1 M_{\odot}$ to $3 M_{\odot}$ (see, e.g., Weber 1999). The predicted initial mass function from su-

Send offprint requests to: D. J. Schwarz, e-mail: dschwarz@hep.itp.tuwien.ac.at pernova simulations provides a more narrow mass range within $1 M_{\odot}$ to $2 M_{\odot}$, depending on the supernova type (Timmes et al. 1996).

The neutron star mass function is of great importance not only for astrophysical purposes, but also for fundamental physics. On the one hand, neutron stars are the only known place in nature where theoretical ideas on the phase diagram of quantum chromodynamics at high density may be tested (for recent reviews see Schwarz 1998, Weber 1999 and Alford et al. 2000). On the other hand, the equation of state of neutron stars is important to predict the wave pattern of gravitational radiation emitted in mergers of neutron star binaries, one of the most important sources expected for gravitational wave interferometers like $\mathrm{LIGO}^{1}$ and $\mathrm{VIRGO}^{2}$.

Gravitational lensing (see, e.g., Schneider et al. 1992) by now has been proven to be a very powerful technique to study mass distributions. For dark or low-luminosity stellar mass objects, gravitational microlensing (for reviews see Paczyński 1996 and Zakharov \& Sazhin 1998) provides a tool to measure their masses. Gravitational microlensing towards the galactic bulge and towards the galactic

\footnotetext{
${ }^{1}$ Laser Interferometer Gravitational Wave Observatory http://www.ligo.caltech.edu/

2 http://www.virgo.infn.it/
} 
spiral arms has been studied by various groups $\left(\mathrm{OGLE}^{3}\right.$, Udalski et al. 1994, 2000; Wozniak et al. 2001; $\mathrm{MACHO}^{4}$, Alcock et al. 1997, 1999, 2000; Popowski et al. 2001; EROS $^{5}$ Derue et al. 1999, 2001). The optical depth of the bulge is $\tau=(1.4 \pm 0.3) \times 10^{-6}$ (Popowski et al. 2001) and drops to $\tau=0.45_{-0.11}^{+0.24} \times 10^{-6}$ (Derue et al. 2001) in the spiral arms. About 800 candidates for gravitational microlensing have been observed.

In this work we show that gravitational microlensing of neutron stars in the galaxy can in principle be observed with present-day techniques (photometric lensing). We find that the rate of observing a neutron star microlensing event is about $10^{-7}$ per year per observed light source in the direction of the galactic bulge; the optical depth is $\sim 10^{-8}$. Correspondingly, if we assume that the pulsar number density is proportional to the neutron star number density, the rate of observing a pulsar as a microlens is lower by a factor $10^{-3}-10^{-4}$. As the number of monitored stars is $\sim 10^{7}$ in each experiment, we expect that no such observation has been made yet. A data comparison between the ATNF Pulsar Catalogue ${ }^{6}$ and known microlensing events towards the galactic bulge and towards the galactic spiral arms (OGLE, Wozniak et al. 2001; MACHO, Alcock et al. 1997, 1999, 2000; EROS, Derue et al. 1999, 2001) shows that indeed none of the known pulsars has been acting as an observed microlens so far.

Once a microlensing event of appropriate duration (a few days to a few months) has been found the challenge is to figure out whether a neutron star or a different compact object has been acting as lens. This task may be accomplished by means of astrometric microlensing (Høg et al. 1995; Miyamoto \& Yoshi 1995; Walker 1995; MiraldaEscudé 1996; Boden et al. 1997). Astrometric lensing measures the shift of the centre of light of the source star. While photometric lensing is most efficient if the source passes through the Einstein ring, astrometric lensing is most efficient if the source passes close to, but outside, the Einstein ring. sThe most important background will be main sequence stars that are not properly resolved (Gould 2000). White or brown dwarfs as well as black holes are candidates for similar microlensing events due to their similar masses. As has been shown by Gould (2000), upcoming astrometric space missions (like GAIA $^{7}$ and $\mathrm{SIM}^{8}$ ) combined with ground-based observations should be able to pick out a handful of neutron stars. The observation of astrometric lensing will also be possible with the help of long-baseline optical interferometry, and has been studied in some detail for the ESO Very Large Telescope

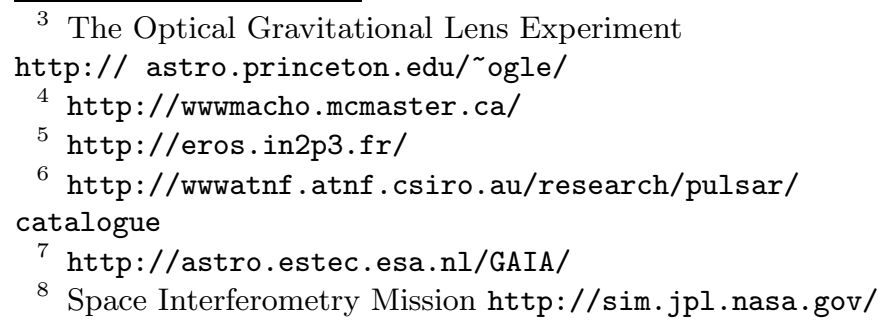

Interferometer $\left(\mathrm{VLTI}^{9}\right)$ by Delplancke et al. (2001). The ESO VLTI offers yet another new possibility; it might be possible for the first time to resolve the double images of microlensed stars. For events that are long enough to observe the parallax effect of the light-curve, a measurement of the lens mass will be possible (Delplancke et al. 2001).

In order to improve the lensing probability for neutron stars we propose a new search strategy that looks for the microlensing event of a known nearby high proper motion pulsar (sweeping a large angle on the sky). In that case the distance and velocity are constrained from the dispersion measure or via parallax measurement and a measurement of the pulsar mass should be possible. Thus gravitational microlensing might enable us to determine the mass of stand-alone pulsars. The cross section of astrometric lensing is larger than that of photometric lensing, which additionally enhances the probability of detection. Thus astrometric measurements around high velocity proper motion pulsars in dense fields might allow a mass determination of pulsars. The potential of forthcoming astrometric space missions for astrometric microlensing of ordinary stars has been investigated in detail by Paczyński (1998), Gould (2000) and Salim \& Gould (2000) for SIM and by Belokurov \& Evans (2001) for GAIA.

In Sect. 2 we use two pulsar population models of our galaxy to estimate the lensing rate and the optical depth towards different directions. In Sect. 3 we describe the catalogue comparison of pulsars and gravitational microlensing events. We suggest a search strategy to increase the probability to find a neutron star that acts as a gravitational microlense in Sect. 4 and end with a short conclusion.

\section{Lensing rate and optical depth}

Let us first investigate the possibility that a neutron star is detected by photometric microlensing.

\subsection{Lensing rate}

A simple estimate for the rate $p$ of gravitational microlensing events per observed background star is obtained easily. We consider a neutron star at distance $r$ that passes by the line of sight to a background star at distance $D_{*}$ within the Einstein radius

$R_{\mathrm{E}}=\sqrt{\frac{2 R_{\mathrm{S}}}{D_{*}} r\left(D_{*}-r\right)}$,

where $R_{\mathrm{S}}$ denotes the Schwarzschild radius. The lensing rate is proportional to the solid angle that is swept by the lens, which depends on the (projected) velocity $v$ and the Einstein radius $R_{\mathrm{E}}$ of the lens. Moreover, the lensing rate is proportional to the distance $D_{*}$ of the source star and to the number density $n$ of lenses (neutron stars), which we take as constant for this simple estimate. Let us for the moment assume that all neutron stars are distributed

\footnotetext{
9 http://www.eso.org/projects/vlti/
} 


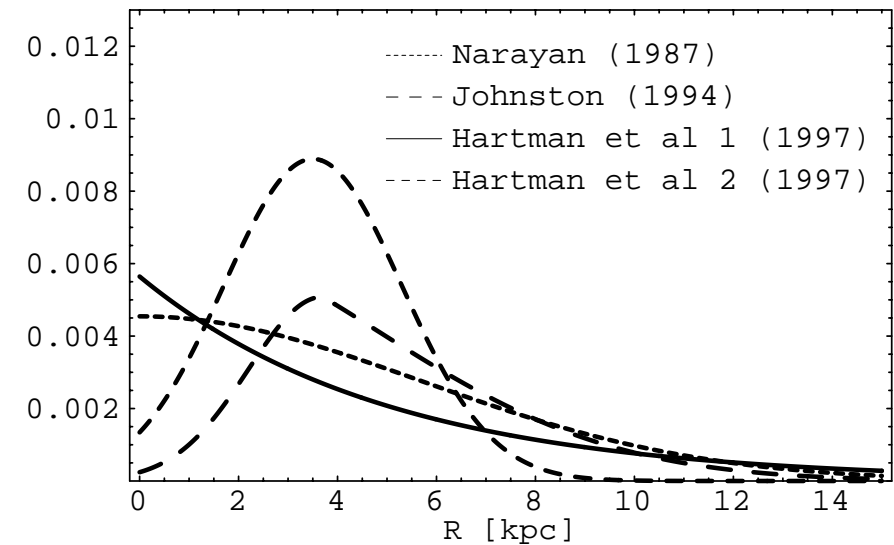

Fig. 1. Four models for the radial distribution of neutron stars in the galactic disk. All four distributions are normalised to unity.

homogeneously in a cylinder with radius $10 \mathrm{kpc}$ and height $1 \mathrm{kpc}$ and that the number of neutron stars in our Galaxy is $N_{\mathrm{NS}}=10^{9}$. By putting $D_{*}=10 \mathrm{kpc}, r=D_{*} / 2, M_{\mathrm{NS}}=$ $1 M_{\odot}$ and $v=200 \mathrm{~km} \mathrm{~s}^{-1}$ we find

$p \sim D_{*} R_{\mathrm{E}} n v \sim 10^{-7} \mathrm{yr}^{-1}$.

Presently, a few tens of millions of stars have been monitored over some years by various collaborations. This suggests that about a handful of by now observed lenses should be neutron stars. This rough estimate is consistent with the estimate given in Gould (2000). The difficulty is to tell which of the observed microlensing events was due to a neutron star. There are only about $10^{5}$ galactic pulsars (Narayan 1987), which gives a pulsar lensing rate of the order $10^{-11} \mathrm{yr}^{-1}$. Typically, a present-day experiment will therefore not find microlensing events that are due to pulsars.

In order to calculate the lensing rate and the optical depth more precisely we have to take into account that the number density of neutron stars is not constant within our galaxy. There are several models for the pulsar number density $n_{\mathrm{PSR}}$ in our galaxy and we assume that the neutron star number density $n$ is proportional to it

$n(R, z)=\frac{N_{\mathrm{NS}}}{N_{\mathrm{PSR}}} n_{\mathrm{PSR}}(R, z)$,

where $R$ is the radial distance of the pulsar/neutron star to the galactic centre in the galactic plane and $z$ is the height.

Following Hartman et al. (1997) we use two different models for the radial dependence of the number density and a model for the $z$-dependence, which was given by Lyne et al. (1998). The two models from Hartman resemble the models from Narayan (1987) and Johnston (1994). Figure 1 shows the number densities of the four models in the galactic plane plotted against the distance from the galactic centre.

We use the assumption (Narayan 1987), that the number density can be factorised

$n(R, z) 2 \pi R \mathrm{~d} R \mathrm{~d} z=N_{\mathrm{NS}}\left[n_{R}(R) 2 \pi R \mathrm{~d} R\right]\left[n_{z}(z) \mathrm{d} z\right]$.
The first model from Hartman is exponential in $R$,

$$
n_{\mathrm{H} 1}(R)=\frac{1}{2 \pi R_{\mathrm{W}}^{2}} \exp \left(-\frac{R}{R_{\mathrm{W}}}\right),
$$

where $R_{\mathrm{W}}=5 \mathrm{kpc}$. There is some evidence for a deficit of pulsars in the galactic centre which was shown by Johnston (1994). The second model from Hartman takes this into account

$n_{\mathrm{H} 2}(R)=\frac{c_{\mathrm{H} 2}}{2 \pi R_{\mathrm{w}}^{2}} \exp \left(-\frac{\left(R-R_{\max }\right)^{2}}{2 R_{\mathrm{w}}^{2}}\right)$,

where $R_{\mathrm{w}}=1.8 \mathrm{kpc}$ and $R_{\max }=3.5 \mathrm{kpc}$. The normalisation constant is $c_{\mathrm{H} 2} \approx 0.204$ for the given choice of $R_{\max }$. For Gaussian distribution around the centre $c_{\mathrm{H} 2}=1$. For the $z$-dependence we use the Gaussian

$n_{z}(z):=\frac{1}{\sqrt{2 \pi} \sigma} \exp \left(-\frac{1}{2} \frac{z^{2}}{\sigma^{2}}\right)$

with $\sigma=0.45 \mathrm{kpc}$ (Lyne et al. 1998).

We transform the Eqs. (5)-(7) into spherical coordinates $(r, \theta, \phi)$ with the sun at the origin via

$z=r \sin \theta$,

$R^{2}=r^{2} \cos ^{2} \theta+R_{\mathrm{SC}}^{2}-2 r R_{\mathrm{SC}} \cos \theta \cos \phi$.

The distance from the sun to the centre of the galaxy is given by $R_{\mathrm{SC}}=8.5 \mathrm{kpc}$.

Hansen \& Phinney (1997) suggested a Maxwell distribution for the kick velocities of neutron stars:

$f(v)=\sqrt{\frac{2}{\pi}} \frac{v^{2}}{\sigma_{\mathrm{v}}^{3}} \exp \left(-\frac{v^{2}}{2 \sigma_{\mathrm{v}}^{2}}\right)$

where $\sigma_{\mathrm{v}}=190 \mathrm{~km} \mathrm{~s}^{-1}$, which corresponds to a mean velocity at birth of $\sim 300 \mathrm{~km} \mathrm{~s}^{-1}$. For pulsars that are older than $10^{7}$ years they find a mean velocity of $198 \pm 53 \mathrm{~km} \mathrm{~s}^{-1}$. We can now calculate the lensing rate, which is given by

$p\left(D_{*}, \theta, \phi\right)=N_{\mathrm{NS}} \pi v_{\perp} \int_{0}^{D_{*}} R_{\mathrm{E}}\left(r, D_{*}\right) n(r, \theta, \phi) \mathrm{d} r$.

It only depends on the part of the velocity vector that lies in the lens plane. Therefore we have to calculate the mean velocity projection on the lens plane $v_{\perp}$ which is given by

$v_{\perp}=\frac{1}{\pi} \int_{-\frac{\pi}{2}}^{\frac{\pi}{2}} v \cos \alpha \mathrm{d} \alpha=\frac{2 v}{\pi}$.

Figures 2 and 3 show the lensing rate as a function of the galactic longitude $\left(\phi \equiv\left(l / 180^{\circ}\right) \pi\right)$ and as a function of the distance to the light source, respectively. It is most promising to search for neutron stars that act as microlense in the direction of the galactic bulge, but it is also reasonable to look into the disk at galactic longitudes below $50^{\circ}$. 


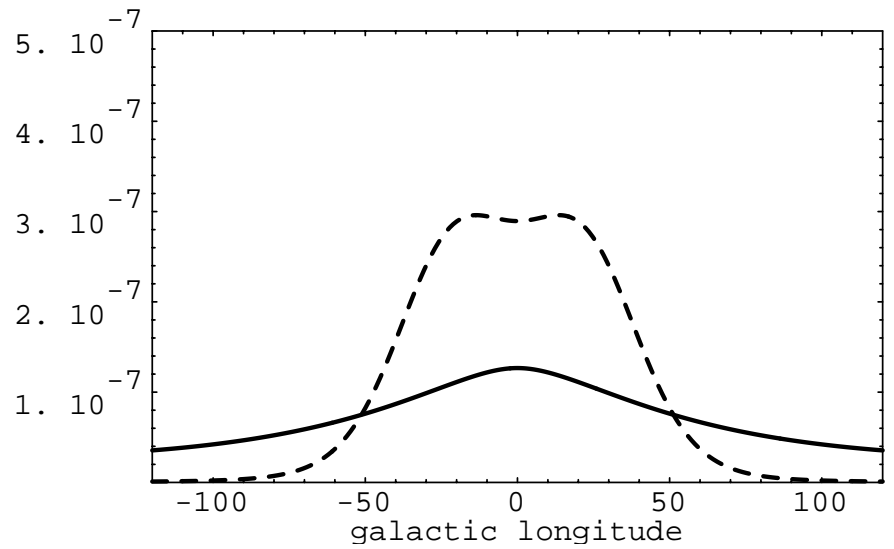

Fig. 2. Lensing rate in units of number of events/source/year for light sources in the galactic plane at a distance of $D_{*}=$ $7.5 \mathrm{kpc}$, as a function of the galactic longitude. The two estimates are based on two models for the radial distribution of pulsars by Hartman et al. (1997). The solid (dashed) line represents the model $\mathrm{H} 1(\mathrm{H} 2)$.

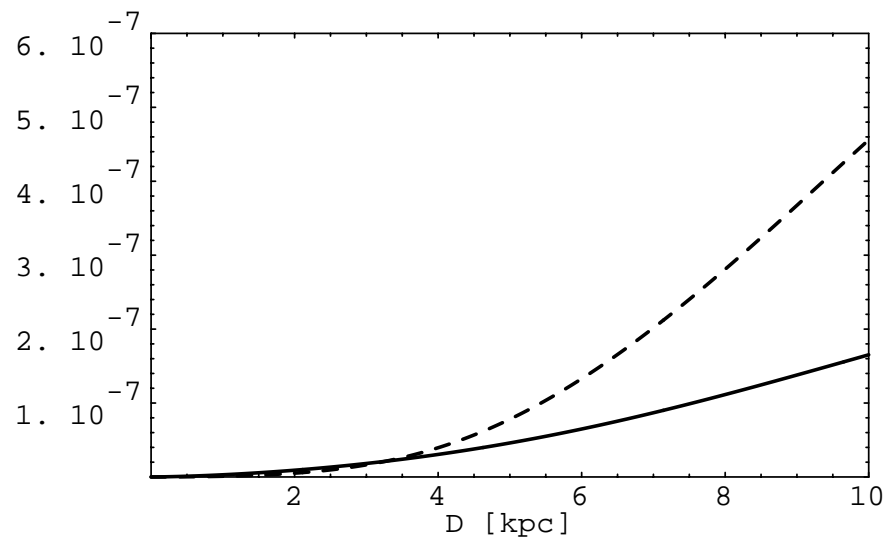

Fig. 3. Lensing rate in units of number of events/source/year for light sources at galactic longitude $30^{\circ}$ in the galactic plane, as a function of distance $D_{*}$, for the same models as in Fig. 2.

\subsection{Optical depth}

Analogous to (2) we estimate the order of the optical depth,

$\tau \sim n D_{*} R_{\mathrm{E}}^{2} \sim \frac{p R_{\mathrm{E}}}{v} \sim p 0.1 \mathrm{yr} \sim 10^{-8}$.

A more thorough estimate for the optical depth is obtained from

$\tau\left(D_{*}, \theta, \phi\right)=\int_{0}^{D_{*}} \pi R_{\mathrm{E}}^{2}\left(r, D_{*}\right) n(r, \theta, \phi) \mathrm{d} r$.

Figures 4 and 5 show the optical depth as a function of the galactic longitude and as a function of the distance to the light source, respectively.

\subsection{Typical timescale}

Consider a lens of mass $M$, passing the line of sight of a distant source at a perpendicular velocity $v_{\perp}$. The typical

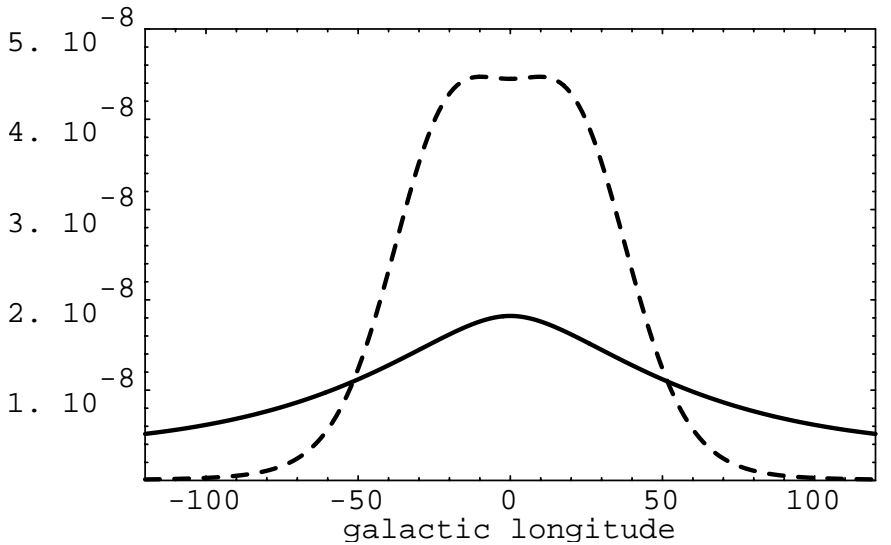

Fig. 4. Optical depth for light sources in the galactic plane at a distance of $D_{*}=7.5 \mathrm{kpc}$, as a function of the galactic longitude, for the same models as in Fig. 2.

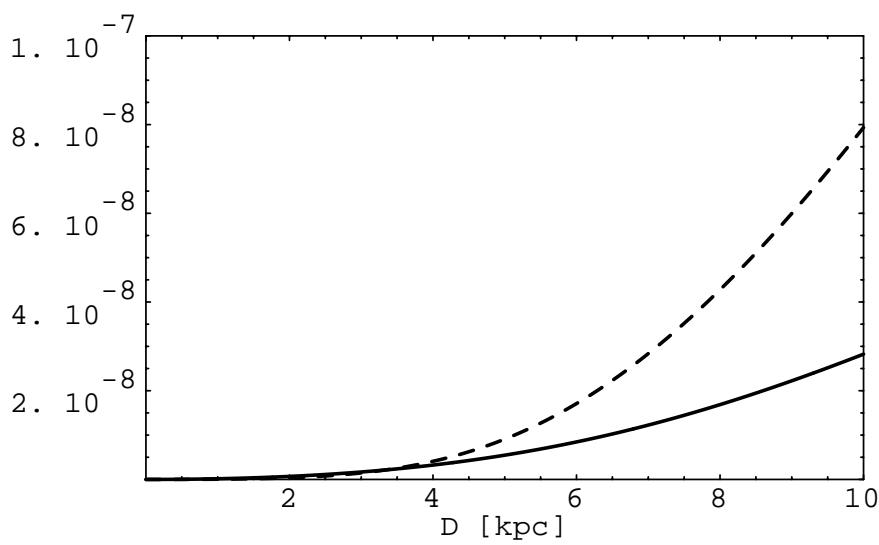

Fig. 5. Optical depth for light sources at galactic longitude $30^{\circ}$ in the galactic plane, as a function of distance $D_{*}$, for the same models as in Fig. 2.

time scale $t_{\text {var }}$ of the microlensing event is given by

$$
\begin{aligned}
t_{\mathrm{var}} & \equiv \frac{R_{\mathrm{E}}}{v_{\perp}} \\
& \approx 25 \mathrm{~d}\left(\frac{M}{M_{\odot}}\right)^{1 / 2}\left(\frac{D}{1 \mathrm{kpc}}\right)^{1 / 2}\left(\frac{200 \mathrm{~km} \mathrm{~s}^{-1}}{v_{\perp}}\right),
\end{aligned}
$$

where $D=r\left(D_{*}-r\right) / D_{*}$. For $M=1.4 M_{\odot}$ we find that the duration of a neutron star microlensing event might range from a few days for close and fast neutron stars up to a few months for neutron stars close to the galactic bulge.

The distribution of timescales is estimated in Fig. 6 . We plot

$$
\begin{aligned}
\frac{\mathrm{d} p\left(t_{\mathrm{var}}\right)}{\mathrm{d} \ln t_{\mathrm{var}}}= & \frac{2 N_{\mathrm{NS}}}{t_{\mathrm{var}}^{2}} \\
& \times \int_{0}^{D_{*}} R_{\mathrm{E}}^{3}\left(r, D_{*}\right) f\left[\frac{R_{\mathrm{E}}\left(r, D_{*}\right)}{t_{\mathrm{var}}}\right] n(r, \theta, \phi) \mathrm{d} r
\end{aligned}
$$

at $\theta=\phi=0$ for $D_{*}=3.25 \mathrm{kpc}$ and $D_{*}=7.5 \mathrm{kpc}$, respectively. We use the velocity distribution (10), since we expect young neutron stars at small scale height. As can be seen from the comparison of the timescale distributions 


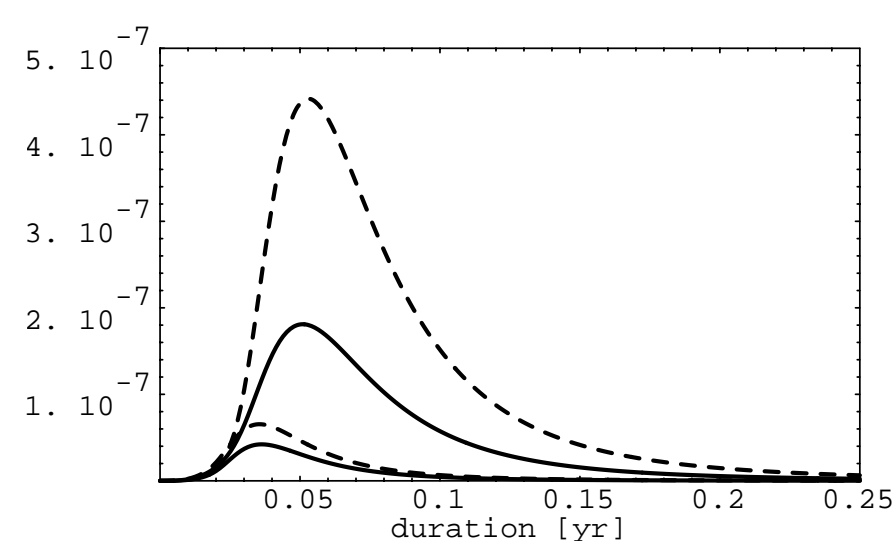

Fig. 6. Timescale distribution towards the galactic centre for light sources at $D_{*}=7.5 \mathrm{kpc}$ (upper lines) and $D_{*}=3.25 \mathrm{kpc}$ (lower lines), for the same models as in Fig. 2.

for sources close to the galactic centre and sources at moderate distances, the width of the timescale distribution is dominated by the sources close to the galactic centre. In both models for the radial distribution the timescale distribution peaks at durations of $\sim 20$ days.

\subsection{Astrometric microlensing}

Astrometric microlensing makes use of the shift of the centroid of the combined (unresolved) images of the light source. While the light amplification used in the photometric effect drops outside the Einstein ring as

$A \sim 1+2\left(\frac{\theta_{\mathrm{E}}}{\theta_{\mathrm{sl}}}\right)^{2}$,

the centroid shift is given by

$\Delta \theta \sim \frac{\theta_{\mathrm{E}}^{2}}{\theta_{\mathrm{sl}}}$

Here $\theta_{\mathrm{E}} \equiv R_{\mathrm{E}} / r$ and $\theta_{\mathrm{sl}}$ is the angle between the source and the lense. For a nearby neutron star we may assume $D_{*} \gg r$, thus $\theta_{\mathrm{E}} \approx 9\left(M / M_{\odot}\right)^{1 / 2}(100 \mathrm{pc} / r)^{1 / 2}$ mas, giving rise to a typical centroid shift of

$\Delta \theta \approx 81\left(\frac{M}{M_{\odot}}\right)\left(\frac{100 \mathrm{pc}}{r}\right)\left(\frac{1^{\prime \prime}}{\theta_{\mathrm{sl}}}\right) \mu \mathrm{as}$.

GAIA will achieve an astrometric accuracy of $11 \mu$ as at $V=15 \mathrm{mag}$, which drops to $160 \mu$ as at $V=20 \mathrm{mag}$ (Perryman et al. 2001), while SIM will resolve $4 \mu$ as for sources as faint as that magnitude (Boden et al. 1997). With the help of VLTI an astrometric accuracy of the order of $10 \mu$ as can be expected (Delplancke et al. 2001) with a limiting magnitude $K=19-20$ (available from the middle of 2004). Thus it will be possible, at least in principle, to measure the centroid shift of background stars separated less than a few "from a nearby neutron star.

For a survey mission like GAIA, the optical depth and timescale of lensing may be estimated as above, simply correcting for the larger cross section due to the astrometric effect. If one adopts an accuracy of $\sim 100 \mu$ as,
Table 1. Catalogue comparison of the coordinates of gravitational microlensing events and radio pulsars.

\begin{tabular}{|l|r|}
\hline collaboration & number of microlensing events \\
\hline OGLE & bulge 520 \\
MACHO & bulge 144 \\
EROS & bulge 19 \\
& spiral arms 7 \\
& total 690 \\
\hline collaboration & number of radio pulsars \\
\hline ATNF & 1308 \\
\hline
\end{tabular}

and aims at measuring the centroid shift with an error of $10 \%, \theta_{\mathrm{sl}} / \theta_{\mathrm{E}}$ might be as big as 10 , thus increasing the cross section from photometric lensing by a factor of 100 and the time scale by a factor of 10 . However, the sampling of GAIA will be sparse compared with ground-based ongoing microlensing experiments and only a small fraction of all astrometric microlensing events will be detected (Belokurov \& Evans 2001).

On the other hand SIM will focus on astrometry of preselected targets. For photometrically-detected microlensing events it will be possible to detect the centroid shift and to obtain high accuracy mass determinations.

\section{Catalogue comparison}

As a consistency check of the above estimates of the photometric lensing rates we investigate whether any pulsar has acted as a microlens thus far.

The positions of 690 candidates for gravitational microlensing events towards the galactic bulge and the spiral arms $\left(\right.$ OGLE $^{10}$, Wozniak et al. 2001; MACHO ${ }^{11}$, Alcock et al. 1997, 1999, 2000; $\operatorname{EROS}^{12}$, Derue et al. 1999, 2001) have been compared with the positions of 1308 radio pulsars from the ATNF Pulsar Catalogue ${ }^{13}$, which is based on the Princeton Pulsar Catalogue ${ }^{14}$ (Taylor et al. 1993), the Parkes Multibeam Pulsar Survey ${ }^{15}$, (Manchester et al. 2001) and the Swinburne Intermediate Latitude Pulsar Survey $^{16}$ (Edwards et al. 2001). Table 1 lists the number of records of the individual groups. Some of the microlensing events have been detected by more than one group. We did not account for this in the table.

All positions are given in the J2000 coordinate system. However, not all of the measurements were made at the same time. Therefore we have to consider a change

\footnotetext{
10 Catalogue of 520 Microlensing Events in the Galactic Bulge:

http://astro.princeton.edu/ wozniak/dia/lens/

11 http://wwwmacho.mcmaster.ca/Data/bulgefts.html

12 alerts 1998-2000, http://www-dapnia.cea.fr/Phys/Spp/ Experiences/EROS/alertes.html

13 http://wwwatnf .atnf .csiro.au/research/pulsar/ catalogue

14 http://pulsar.princeton.edu/pulsar/catalog.shtml

15 http://wwwatnf .atnf .csiro.au/research/pulsar/ pmsurv/

16 http://astronomy.swin.edu.au/pulsar/
} 
Table 2. All pulsars with measured proper motion $|\mu|>100$ mas/yr and $\left|\Delta \mu_{\mathrm{ra}, \mathrm{dec}}\right|<10$ mas/yr from the Princeton Pulsar Catalogue. Coordinates are given in the J2000 system at the specified epoch. For catalogue entries without position epoch we quote the period epoch of the respective pulsar. The distance estimates are based on the dispersion measure. The number of objects within $15^{\prime \prime}$ of a pulsar is obtained with the help of VizieR from the USNO-A2.0/GSC2.2.1 catalogues.

\begin{tabular}{|c|c|c|c|c|c|c|c|c|c|c|}
\hline PSR J & $\begin{array}{r}\mu_{\mathrm{ra}} \\
\mathrm{mas} / \mathrm{yr}\end{array}$ & $\begin{array}{r}\mu_{\mathrm{dec}} \\
\mathrm{mas} / \mathrm{yr}\end{array}$ & $\begin{array}{r}|\mu| \\
\operatorname{mas} / \mathrm{yr}\end{array}$ & $\begin{array}{r}\mathrm{ra} \\
\mathrm{h}: \mathrm{m}: \mathrm{s}\end{array}$ & $\begin{array}{r}\operatorname{dec} \\
\circ \vdots^{\prime \prime}\end{array}$ & $l$ & $\begin{array}{l}b \\
\end{array}$ & $\begin{array}{r}\text { epoch } \\
\text { yr }\end{array}$ & $\begin{array}{r}r \\
\mathrm{kpc}\end{array}$ & $\begin{array}{r}\text { sources } \\
\theta_{\mathrm{sl}}<15^{\prime \prime}\end{array}$ \\
\hline $0437-4715$ & $114 \pm 2$ & $-72 \pm 4$ & 106 & $04: 37: 15.71$ & $-47: 15: 07.0$ & 253 & -42 & 1993 & 0.14 & $1 / 0$ \\
\hline $0826+2637$ & $61 \pm 3$ & $-90 \pm 2$ & 105 & 08:26:51.31 & $+26: 37: 25.6$ & 172 & 35 & 1969 & 1.25 & $0 / 0$ \\
\hline $1136+1551$ & $-102 \pm 5$ & $357 \pm 3$ & 370 & 11:36:03.30 & $+15: 51: 00.7$ & 240 & 69 & 1975 & 0.27 & $0 / 0$ \\
\hline $1239+2453$ & $-106 \pm 4$ & $42 \pm 3$ & 105 & $12: 39: 40.47$ & $+24: 53: 49.3$ & 252 & 87 & 1985 & 0.56 & $0 / 0$ \\
\hline $1932+1059$ & $99 \pm 6$ & $39 \pm 4$ & 105 & 19:32:13.90 & $+10: 59: 32.0$ & 47 & -4 & 1991 & 0.17 & $4 / 5$ \\
\hline $2225+6535$ & $144 \pm 3$ & $112 \pm 3$ & 127 & $22: 25: 52.36$ & $+65: 35: 33.8$ & 109 & 7 & 1991 & 2.00 & $1 / 1$ \\
\hline
\end{tabular}

of the pulsar position that will lead to a deviation angle $\alpha \approx v t / r$, where $t$ is the time between the pulsar detection and the microlensing event, $v_{\perp}$ is the (projected) pulsar velocity and $r$ the pulsar distance. For a conservative estimate that includes pulsars that are close, fast, and have been detected long ago, we take $r=100 \mathrm{pc}$, $v=500 \mathrm{kms}^{-1}, t=10 \mathrm{yrs}$, which gives $\alpha \approx 10^{\prime \prime}$. We found no candidate pair with an angular separation below this value. In a second step we looked for the closest pair, which is PSR J1807-2715 and the OGLE microlensing event sc18 2757. The angular separation is $0.08 \mathrm{deg}$. According to the Priceton Pulsar Catalogue PSR J18072715 is at an estimated distance of $9.67 \mathrm{kpc}$ and no proper motion is reported. Thus it is highly unlikely that it can be responsible for the OGLE event sc18 2757. These findings are in agreement with our estimate, which predicts an extremely small lensing rate for pulsars, i.e. $\sim 10^{-4} / \mathrm{yr}$ for $10^{7}$ monitored stars.

\section{A new search strategy}

We have shown that probably some of the gravitational microlensing events towards the galactic bulge are due to neutron stars. For a specific microlensing event with a duration of a few days to a few months, one is interested in finding out whether the lens is a neutron star or a different object. There is not very much hope that with present day experiments a microlensing event will be detected whose lens is a known pulsar. Our catalogue comparison has confirmed this result. To raise the low probability it is clear that a better search strategy is needed. With the prospects of astrometric lensing, we can expect that high precision measurements of masses will become possible, but still the problem of identifying the nature of the lense will remain, especially for masses of $1-3 M_{\odot}$, since many possible objects are stable in that mass range.

A unique identifier of a neutron star is the possibility that it is observed as a radio pulsar. Instead of choosing a field of background stars we suggest to choose a pulsar and to monitor the background stars around it. The solid angle $\Omega$ that is swept during $\Delta t$ by the Einstein-ring of a moving Schwarzschild-lens is given by

$\Omega=\frac{1}{4 \pi r^{2}}\left(\pi R_{\mathrm{E}}^{2}+2 R_{\mathrm{E}} v_{\perp} \Delta t\right) \simeq \frac{R_{\mathrm{S}}}{2 r}\left(1+\frac{2 \Delta t}{\pi t_{\mathrm{var}}}\right)$, for $r \ll D_{*}$. Our strategy therefore must be to observe distant background stars in the direction of a close, fastmoving neutron star. Table 2 shows some high proper motion pulsars from the Princeton Pulsar Catalogue. We include all pulsars whose proper motion have been measured to be $|\mu|>100$ mas/yr and keep only those with proper motion estimates better than $10 \%$. We expect that pulsars at low galactic latitude have the highest probablility of acting as a gravitational lens. This expectation is confirmed by the result of a search for appropriate background stars, based on the USNO-A2.0 (Monet et al. 1998) and GSC2.2.1 ${ }^{17}$ catalogues. These catalogues contain 526, 280, 881 resp. 455, 851, 237 sources and both cover the entire sky. The USNO-A2.0 catalogue above declination $-30^{\circ}$ (only those plates are relevant in our context, see below) goes as deep as 21 in $O$ (blue plates) and 20 in $E$ (red plates), whereas the GSC2.2.1 catalogue is limited by $F=18.5$ in the red and $J=19.5$ in the blue. We search in a radius of $15^{\prime \prime}$. The number of hits are shown in the last column of Table 2 for USNO-A2.0/GSC2.2.1. This procedure selects three pulsars as interesting candidates for lensing.

The only candidate at high galactic latitude, PSR J0437-4715, is a binary pulsar and the USNO-A2.0 object 0375-01566415 (epoch 1981) is separated by only $\approx 0.5^{\prime \prime}$ after the measured pulsar proper motion is used to extrapolate to epoch 1981. Thus the USNO object seems to be identical with the binary companion identified by Bell et al. (1993), probably a white dwarf with $R=20.1$, since they find no other star brighter than $R=23.8$ within $6^{\prime \prime}$ of the radio position.

The closest object (USNO-A2.0 object 1500-09063417, GSC2.2.1 object N013123248640) to PSR J2225+6535 is separated by $14.7^{\prime \prime}$ and thus is too far away from the pulsar for significant lensing.

The most promising candidate is the pulsar PSR J1932+1059 (B1929+10). This pulsar is at a (dispersion measure) distance of about $0.17 \mathrm{kpc}$ (Princeton Pulsar Catalogue) and moves 105 mas/yr. It lies towards the galactic disk $\left(l=47.4^{\circ}, b=-3.9^{\circ}\right)$ and is found to have 7 objects brighter than red magnitude $\sim 20$ within $15^{\prime \prime}$. From the 9 hits shown in Table 3, 2 objects are

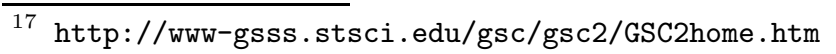


Table 3. Objects from the USNO-A2.0 and GSC2.2.1 catalogues within $15^{\prime \prime}$ of PSR J1932+1059 at position epoch $1991 . x$ and $y$ denote the relative positions to the pulsar in the arc projection. The astrometric (photometric) accuracy of the USNO-A catalogue is typically $0.25^{\prime \prime}(0.25 \mathrm{mag})$. The astrometric accuracy of GSC2.2 is typically $0.3^{\prime \prime}$, the photometric errors are included in the table. We refer to the objects in the text by the number in the last column of the table. Objects 1 and 2 seem both to split into two objects ( $5 \& 6$ and $7 \& 8$ resp.) in GSC2.2. Objects 3 and 4 are both beyond the magnitude limit of GSC2.2, object 9 does not have a counterpart in USNO-A2. Thus we find 7 objects in total.

\begin{tabular}{|l|l|l|l|l|l|l|}
\hline USNO-A2.0 & $x\left[^{{ }^{\prime \prime}}\right]$ & $y\left[^{\prime \prime}\right]$ & blue[mag] & red[mag] & epoch[yr] & id \\
\hline $0975-15208581$ & -1.58 & -3.91 & 15.0 & 13.2 & 1954 & 1 \\
$0975-15209296$ & 4.68 & 5.04 & 19.3 & 17.7 & 1954 & 2 \\
$0975-15208295$ & -3.96 & 7.60 & 20.2 & 19.6 & 1954 & 3 \\
$0975-15207539$ & -10.23 & 6.01 & 20.2 & 19.5 & 1954 & 4 \\
\hline GSC2.2.1 & $x\left[^{\prime \prime}\right]$ & $y\left[^{\prime \prime}\right]$ & blue $[\mathrm{mag}]$ & red[mag] & epoch[yr] & id \\
\hline N0232103208857 & -2.33 & -3.06 & & $14.22 \pm 0.40$ & 1993 & $5(1 ?)$ \\
N0232103780 & -1.93 & -4.66 & $14.85 \pm 0.42$ & $13.44 \pm 0.40$ & 1993 & $6(1 ?)$ \\
N023210364008 & 4.66 & 4.86 & & $17.45 \pm 0.44$ & 1992 & $7(2 ?)$ \\
N0232103208858 & 4.82 & 5.08 & & $18.10 \pm 0.41$ & 1993 & $8(2 ?)$ \\
N023210363839 & -11.30 & -5.29 & $18.68 \pm 0.42$ & $17.11 \pm 0.44$ & 1992 & 9 \\
\hline
\end{tabular}

counted twice (see caption of table). In Fig. 7 (top panel) we map 9" around the pulsars radio position in 1991. The crosses indicate its estimated position in the years 2001, 2011 and 2021. The pulsar moves away from the objects 1, 5, 6 and towards 2, 7, 8 and remains at an almost constant distance to 3. At epoch 2001 (2011, 2021) objects 5 and 7 are separated from the pulsar by $4.8^{\prime \prime}$ $\left(5.7^{\prime \prime}, 6.8^{\prime \prime}\right)$ and $5.9^{\prime \prime}\left(4.9^{\prime \prime}, 4.0^{\prime \prime}\right)$, respectively.

In 1994, Pavlov et al. (1996) identified the optical counterpart of PSR J1932+1059 using the Hubble Space Telescope. The offset from the estimated radio position at epoch 1994.5 was $0.39^{\prime \prime}$. As a by-product they found 6 additional stars within $4^{\prime \prime}$ of the radio position. We show their positions (from Table 5 of Pavlov et al. 1996) in the bottom panel of Fig. 7, which is a magnification of the top panel by a factor of 2 . The pulsar is almost in the middle between HST objects 1 and 2 and approaches HST object 5. At 2010 (2015) the pulsar will be separated from HST objects 1,2 and 5 by $2.66^{\prime \prime}\left(3.16^{\prime \prime}\right), 1.74^{\prime \prime}\left(1.95^{\prime \prime}\right)$ and $1.81^{\prime \prime}\left(1.35^{\prime \prime}\right)$, respectively. According to Pavlov et al. (1996), objects $1-3,5$ and 6 are perhaps very distant white dwarfs strongly reddened by interstellar extinction.

The angular scale of the Einstein radius of PSR J1932+1059 is 8.2 mas, thus there is no photometric lensing effect. However, there is a chance to detect the astrometric effect. Let us first consider the possibility of detecting lensing with the help of the objects from Table 3. These objects are considerably brighter than the six HST objects and therefore astrometric measurements should be simpler. According to Eq. (17) the images of 5 and 7 move between 2010 and 2020 by $2 \mu$ as and $3 \mu$ as. The pulsar and the objects 5 and 7 are to a good approximation on a straight line; the centroid shift is directed along the same line. The accuracy of SIM will be about $4 \mu \mathrm{as}$, thus the effect seems to be just below what can be detected.

Turning to the HST objects the numbers are much more encouraging. Now the pulsar moves away from 1 towards 5 and stays at an almost constant distance to 2 . Between 2010 and 2015 the image of 1 will move away from the pulsar by $4 \mu$ as, the image of 2 will move by $11 \mu$ as almost parallel to the proper motion of the pulsar and the image of 5 will move by $12 \mu$ as towards the pulsar. Thus the angular separation between 1 and 5 should decrease by $8 \mu \mathrm{as}$. If SIM is able to resolve the rather faint stars 1, 2 and 5 a measurement of the astrometric lensing of a pulsar should be possible. The best strategy would be to observe the field a few times per year during the lifetime of SIM.

In the above estimates the uncertainties from the distance estimate are most crucial. We used a distance of 170 pc based on the dispersion measure. This is not very reliable due to inhomogeneities within the interstellar medium. Several attempts to measure the parallax of PSR J1932+1059 gave rise to a confusing situation. Salter et al. (1979) obtained a parallax distance of 3080 pc, while Backer \& Sramek (1982) found a lower limit of 250 pc. Allowing for the range $30-300$ pc implies an uncertainty of an order of magnitude for the predicted image shifts.

\section{Conclusions}

We have argued that measuring the mass of stand-alone pulsars will be possible in the near future by means of gravitational microlensing.

The same strategy could also be used to measure the mass of isolated neutron stars, which are not seen as radio pulsars. Isolated neutron stars (for recent reviews see Treves et al. 2000 and Popov et al. 2000) may be detected as soft X-ray sources due to accretion in the case of old neutron stars or due to energy released in the cooling of young neutron stars. Several candidates have been found by the ROSAT ${ }^{18}$ satellite. For two of those candidates, possible optical counterparts have been identified (RX J185635-3754 by Walter \& Mathews 1997 and RX J0720-3125 by Kulkarni \& van Kerkwijk 1998), which makes them especially interesting. After the submission

\footnotetext{
18 http://wave.xray.mpe.mpg.de/rosat
} 

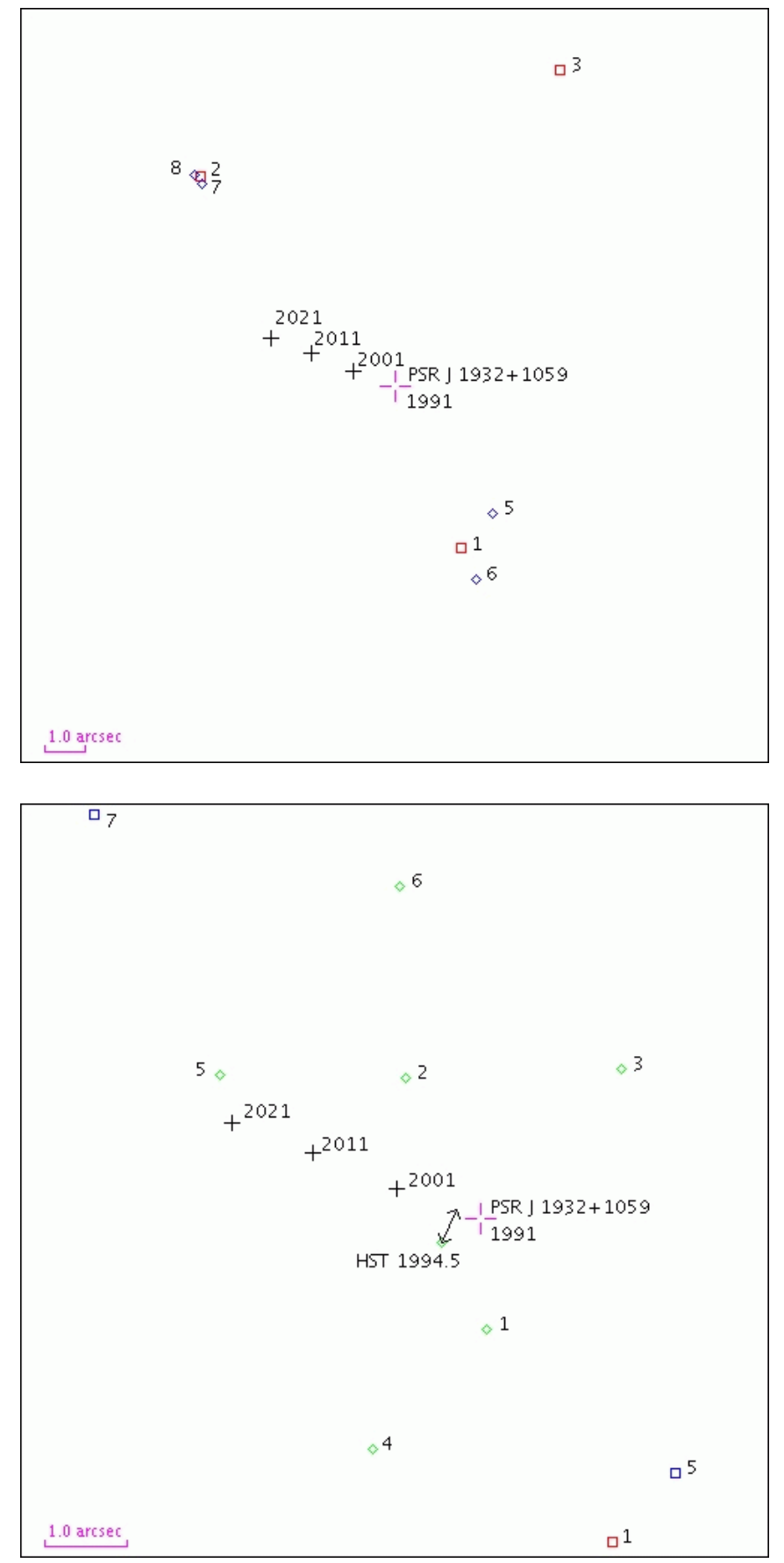

Fig. 7. Top panel: objects from USNO-A2.0 (squares) and GSC2.2.1 (diamonds) within 9" from the 1991 position of PSR J1932+1059. We show also the estimated positions of PSR J1932+1059 for 2001, 2011 and 2021. Bottom panel: a magnification showing the positions of 6 stars and of the pulsar image (diamonds) as seen by the HST (Pavlov et al. 1996). The USNO-A2.0 and GSC2.2.1 objects are now both shown as squares. The arrow shows the difference between the HST image and the estimated radio position at epoch 1994.5. The figures have been created with help of the Aladin Interactive Sky Atlas.

of the first version of this work, Paczyński (2001) pointed out that astrometric lensing of RX J185635-3754 might be possible with the HST in 2003.
In order to increase the list of possible candidates it is important to obtain as many proper motion and parallax measurements of pulsars and isolated neutron stars as possible. Candidates towards the galactic bulge should be especially considered with priority, since the chance of finding a large number of sources for lensing is maximised in that way.

We conclude that measuring neutron star (pulsar) masses by means of gravitational microlensing is possible. We suggest to observe PSR J1932+1059 and three stars around it with SIM over several years to obtain such a mass measurement.

Note added in proof: We learned after acceptance of this work that the possible mass determination of isolated pulsars by gravitational lensing has also been investigated in J. E. Horvath, 1996, MNRAS 278, L46. Recently, a new parallax distance to PSR J1932+1059, $r=331 \pm 10 \mathrm{kpc}$, has been published by W. F. Brisken et al., [astro-ph/0204105]; thus the astrometric effect is a factor of 2 smaller than estimated in this work. Still, it should be possible to detect the astrometric lensing of PSR J1932+1059 with SIM.

Acknowledgements. We would like to thank W. Kegel, D. Lorimer, B. Paczyński and F. Weber for very helpful conversations and references to the literature. This research has made use of the VizieR catalogue access tool and the Aladin Interactive Sky Atlas, CDS, Strasbourg, France. D. J. S. would like to thank the Austrian Academy of Sciences for financial support.

\section{References}

Alcock, C., et al. 1997, ApJ, 479, 119

Alcock, C., et al. 1999, ApJS, 124, 171

Alcock, C., et al. 2000, ApJ, 541, 734; 2001, ApJ, 557, 1035 (Erratum)

Alford, M., Bowers, J. A., \& Rajagopal, K. 2000 [hep-ph/0009357]

Ash, T. D. C., et al. 1999, MNRAS, 307, 357

Backer, D. C., \& Sramek, R. A. 1982, ApJ, 260, 512

Bell, J. F., Bailes, M., \& Bessell, M. S. 1993, Nature, 364, 603

Belokurov, V. A., \& Evans, N. W. 2001 [astro-ph/0112243]

Boden, A. F., Shao, M., \& Van Buren, D. 1997, ApJ, 502, 538

Boden, A., Unwin, S., \& Shao, M. 1997, Proceedings of the ESA Symposium 'Hipparcos - Venice '97', ESA SP-402, 789

Delplancke, F., Górski, K. M., \& Richichi, A. 2001, A\&A, 375, 701

Derue, F., et al. 1999, A\&A, 351, 87

Edwards, R. T., et al. 2001, MNRAS, 326, 358

Derue, F., et al. 2001, A\&A, 373, 126

Gould, A. 2000, ApJ, 535, 928

Hartman, J. W., et al. 1997, A\&A, 322, 477

Hansen, B. M. S., \& Phinney, E. S. 1997, MNRAS, 291, 569

Heap, S. R., \& Corcoran, M. F. 1992, ApJ, 387, 340

Høg, E., Novikov, I. D., \& Polnarev, A. G. 1995, A\&A, 294, 287

Johnston, S. 1994, MNRAS, 268, 595

Kulkarni, S. R., \& van Kerwijk, M. H. 1998, ApJ, 507, L49

Lyne, A. G., et al. 1998, MNRAS, 295, 743 
Manchester, R. N., et al. 2001, MNRAS, 328, 17

Miralda-Escudé, J. 1996, ApJ, 470, L113

Miyamoto, M., \& Yoshi, Y. 1995, AJ, 110, 1427

Monet, D., et al. 1998, U.S. Naval Observatory Flagstaff Station (USNOFS) and Universities Space Research Association (USRA) stationed at USNOFS

Nagase, F. 1989, PASJ, 41, 1

Narayan, R. 1987, ApJ, 319, 162

Paczyński, B. 1996, ARA\&A, 34, 419

Paczyński, B. 1998, ApJ, 494, L23

Paczyński, B. 2001 [astro-ph/0107443]

Pavlov, G. G., Stringfellow, G. S., \& Cordova, F. A. 1996, ApJ, 467,370

Perryman, M. A. C., et al. 2001, A\&A, 369, 339

Popov, S. B., et al. 2000 [astro-ph/0008199]

Popowski, P., et al. 2002 [astro-ph/0202502]

Reynolds, A. P., Bell, S. A., \& Hilditch, R. W. 1992, MNRAS, 256, 631

Reynolds, A. P., et al. 1997, MNRAS, 288, 43

Salim, S., \& Gould, A. 2000, ApJ, 539, 241S

Schneider, P., Ehlers, J., \& Falco, E. E. 1992, Gravitational Lenses (Springer Verlag, Berlin)
Schwarz, D. J. 1998, Nucl. Phys., A642, 336c

Salter, M. J., Lyne, A. G., \& Anderson, B. 1979, Nature, 280, 461

Taylor, J. H., Manchester, R. N., \& Lyne, A. G. 1993, ApJS, 88,529

Thorsett, S. E., et al. 1993, ApJ, 405, L29

Thorsett, S. E., \& Chakrabarty, D. 1999, ApJ, 512, 288

Timmes, F. X., Woosley, S. E., \& Weaver, T. A. 1996, ApJ, 457,834

Treves, A., Turolla, R., Zane, S., \& Colpi, M. 2000, PASP, 112, 297

Udalski, A., et al. 1994, Acta Astron., 44, 165

Udalski, A., et al. 2000, Acta Astron., 50, 1

Walter, F., \& Matthews, L. D. 1997, Nature, 389, 358

Walker, M. A. 1995, ApJ, 453, 37

Weber, F. 1999, Pulsars as Astrophysical Laboratories for Nuclear and Particle Physics (IOP Publishing, Bristol)

Wozniak, P. R., et al. 2001, Acta Astron., 51, 175

Zakharov, A. F., \& Sazhin, M. V. 1998, Physics-Uspekhi, 41, 945 